Ann. Zootech., I977, 26 (4), 547-563.

\title{
Valeur nutritionnelle pour le poulet en croissance, de cinq amylacés tropicaux en relation avec quelques caractéristiques physico-chimiques de leur amidon
}

\author{
O. SZYLIT (*), I. P. BORGIDA, H. BEWA (*), R. CHARBONNIERE (***) \\ et J. DELORT-IAAVAL $\left({ }^{* *}\right)$ \\ (*) Laboratoive d'Écologie Microbienne \\ Contre national de Recherches zootechniques, I.N.R.A., \\ $7835^{\circ}$ Jouy-en-Josas, (France) \\ (**) Laboratoire de Technologie des Aliments des Animaux, I.N.R.A., \\ Chemin de la Géraudière, 44072 Nantes Cedex (France) \\ (***) Laboratoire de Technologie Alimentaive, I.N.R.A. \\ Cerdia, 91305 Massy (France)
}

\begin{abstract}
Résumé
Une expérience poursuivie sur des poulets en croissance (souche I-99) a eu pour but d'évaluer l'efficacité alimentaire de 5 tubercules tropicaux en fonction des caractéristiques physico-chimiques de leur amidon. Le manioc, le taro et l'igname dumetorum ont en commun, des grains d'amidon de faible diamètre (I à I $2 \mu \mathrm{m}$ ), pauvres en amylose ( 8 à I 7 p. cent) et un spectre de diffraction aux rayons $X$ de type $A$ qui les apparente aux amidons de céréales. Le Canna et l'igname cayenensis ont des grains d'amidon beaucoup plus gros $(60$ à $75 \mu \mathrm{m})$, un taux d'amylose élevé (26 p. cent) et un spectre de type $B$ comme celui de la fécule de pomme de terre.

La sensibilité in vitro de ces amidons à l'action de $l^{\prime} \alpha$-amylase de $B$. subtilis décroît dans l'ordre suivant : manioc-igname dumetorum-taro-Canna-igname cayenensis.

Pour les poulets recevant, entre 3 et 6 semaines, les tubercules incorporés dans des régimes isoprotéiques et isoamidon, on observe avec les quatre premiers régimes des gains de poids moyens peu différents (respectivement $40,36,37$ et $36 \mathrm{~g} /$ jour) mais des consommations journalières variables $(82,83,99$ et $9 \mathrm{I} \mathrm{g} / \mathrm{jour})$. Le régime à base d'igname cayenensis donne la plus mauvaise croissance (I $2 \mathrm{~g} /$ jour) et est le moins bien consommé ( $64 \mathrm{~g} /$ jour).

Les amidons de type $A$ sont tous totalement digérés ( $98 \mathrm{p}$. cent). Les amidons de type $\mathrm{B}$ sont moins bien utilisés ( $90 \mathrm{p}$. cent pour Ie Canna; $69 \mathrm{p}$. cent pour l'igname cayenensis). Il en découle des différences au niveau du coefficient d'utilisation pratique de l'azote des régimes.

En conclusion, aucun des paramètres physico-chimiques ou enzymatiques que nous avons considérés, ne peut, à lui seul, constituer un critère de classement de la valeur nutritionnelle d'un amidon. Cependant, une petite taille des grains, un faible taux d'amylose, un spectre de diffraction de type A sont des éléments favorables à une bonne utilisation digestive de l'amidon. Il apparaît de plus que l'efficacité alimentaire (ingéré/gain de poids) du tubercule est plus spécialement en liaison avec la vitesse d'amylolyse in vitro de l'amidon.
\end{abstract}




\section{Introduction}

L'utilisation nutritionnelle d'un amidon natif varie dans le même sens que sa sensibilité in vitro à l'action de l'amylase de $B$. subtilis. Ainsi, il est bien connu que l'amidon de pomme de terre crue, peu sensible à l'action d'alpha-amylases bactériennes (BAKER et al., I950) est également peu digestible en l'état par le poulet (Masson, I954) et les vrais monogastriques (LANGWor'thy et DeUEL, I920; Zelter, Charleti-Lery et Delort-Laval, ig66). Plis récemment, il a été observé qu'un amidon d'amylomaïs, également peu sensible in vitro aux mêmes amylases, est aussi mal utilisé par le poulet (Szylit, Delort-Laval et Borgida, I974).

Or, la dégradation alpha-amylasique du grain d'amidon dépend étroitement de l'accessibilité de ses régions amorphes aux enzymes et de l'importance des liaisons hydrogène interchaînes qui assurent la cohésion des régions cristallines (Mercier, I968). Il semble donc que l'on puisse invoquer comme caractéristiques physiques facilement accessibles à l'analyse et susceptibles d'intervenir sur l'utili. sation nutritionnelle d'un amidon, la taille des grains, les proportions respectives de chaînes anhydroglucose linéaires et ramifiées qui les constituent, l'organisation de leur fraction cristalline.

Les relations de chacune de ces caractéristiques avec l'efficacité nutritionnelle d'un amidon ne sont pas clairement établies. C'est ainsi que les amidons de pomme de terre et de maïs riches en amylose, reconnus peu digestibles par le poulet, sont cependant constitués de grains dont la forme, la taille et la teneur en amylose sont très différentes. Par contre, leur organisation cristalline est la même, pour autant que permette d'en juger l'identité de leurs spectres de diffraction, de type $\mathrm{B}$ dans les deux cas.

D'autre part, les résultats de l'utilisation nutritionnelle d'un même amylacé apparaissent parfois contradictoires. C'est notamment le cas pour le manioc, dont la farine est la plus utilisée en alimentation animale et passe pour permettre une bonne croissance des poulets (Sourss et WIr,D, I95I; I)ictionnaire des aliments pour animaux, Ig65). Pourtant, d'après une revue bibliographique récente (N.A.R., 1974), des doutes subsistent quant à l'efficacité de cet aliment. Ainsi pour certains auteurs (Taboyoyovg, I935; 'Torres, I935; Vogt et STUTE, I963), le manioc est mal utilisé par le poulet et ne peut remplacer le maïs qu'au taux de ro p. cent, alors que pour d'autres (PALISSE et BaraTOU, I973, la substitution du manioc ou de la patate douce à $30 \mathrm{p}$. cent de blé ou de maîs, n'a pas de répercussion sur sa croissance. On peut toutefois se demander dans quelle mesure les caractéristiques propres de l'amidon natif de manioc interviennent seules dans les résultats rapportés, car d'éventuelles altérations de ses grains sont susceptibles de se produire au cours de la récolte et de la fabrication de l'aliment et modifier la valeur alimentaire (Atrinkpahoun, 1972). De plus, la variété utilisée, non spécifiée, pourrait également jouer un rôle.

Afin d'essayer de préciser les rapports entre les caractéristiques mentionnées et la valeur nutritionnelle de régimes différant par l'origine des amylacés qui les constituent, nous avons étudié la croissance de poulets nourris de régimes à base de tubercules amylacés de végétaux divers : manioc (Manihot utilissima), igname (Dioscorea sp.), taro (Colocasia antiquorum), Canna (Canna edulis). Bien que, dans le cas du manioc, il s'agisse d'une racine et non d'un tubercule, ce dernier terme sera cependant employé dans tous les cas pour raison de simplification.

Les ignames cultivées comprennent de nombreuses espèces et variétés, de 
sorte que leurs tubercules peuvent avoir des compositions glucidiques et protidiques variables (Splitstoesser, Martin et Rhodes, I973). Mais tous possèdent des protéines riches en acides aminés indispensables, les soufrés excepté, et des taux de matières azotées qui sont loin d'être négligeables ( 6 à 13 p. cent), d'où leur emploi habituellement réservé à l'alimentation humaine. Dans le présent travail, les tubercules d'ignames retenus sont ceux de Dioscorea dumetorum et Dioscorea cayenensis.

Les tubercules de taro ne servent également qu'à l'alimentation humaine. Par contre, ceux de canna ont été expérimentés avec de bons résultats sur les bovins et les porcs (Szabuniewicz, I953; LE Dividich (*).

En dehors des quelques utilisations citées, 1a littérature ne fournit pratiquement pas de données sur la valeur nutritionnelle de ces divers tubercules pour la volaille.

\section{Matériel et méthodes}

\section{I. - Origine des tubercules utilisés} et mode de préparation des régimes alimentaires

Les tubercules d'ignames sont récoltés au Cameroun et ceux de taro et de canna à la Guadeloupe : ils sont coupés en cossettes qui sont ensuite séchées à 1'air chaud $\left(70^{\circ}\right)$ pendant une nuit. Les " tubercules " de manioc proviennent du Malawi et sont fournis industriellement sous forme de cossettes séchées.

Les régimes sont préparés en incorporant les cossettes sèches et broyées à un aliment équilibré en tenant compte de leurs teneurs en amidon, en azote total et de leur composition en acides aminés (tabl. I), déterminées respectivement suivant les méthodes d'Ewers (I972), de KJeldahl et de Moore, Spackmann et Stein ( $195^{8}$ ). Ces régimes (tabl. 2) ont été fournis aux animaux sous forme de granulés de $2,5 \mathrm{~mm}$ de diamètre et d'environ $\mathbf{I} \mathrm{cm}$ de longueur, obtenus par pressage à froid des mélanges à travers une filière, en veillant à ce que la température n'excède jamais $60^{\circ} \mathrm{C}$.

\section{II. - Caractérisation des amidons}

L'amiclon de chaque tubercule a été caractérisé par :

- son aspect morphologique (taille et forme des grains), déterminé par examens de microscopie photonique effectués en lumière normale et polarisée sur les grains en suspension alcoolique;

- son spectre de diffraction de rayons $\mathrm{X}$, réalisé selon une méthode déjà décrite (ChARBONniÈre et al., I968);

- sa teneur en amylose, déterminée par la méthode de titration ampérométrique de BEMILLER (I964);

- sa sensibilité à une amylolyse in vitro effectuée selon la méthode de MERCIER (I968) et conformément à un protocole déjà décrit (Szyi,IT, DELORT-LAVAL et BORGIDA, I974).

$\left.{ }^{*}\right)$ Communication personnelle. 
TABLEAU I

Composition en acides aminés, matières azolées totales et amidon des tubercules tropicaux Amino acid, crude protein and starch composition of some tropical tubers

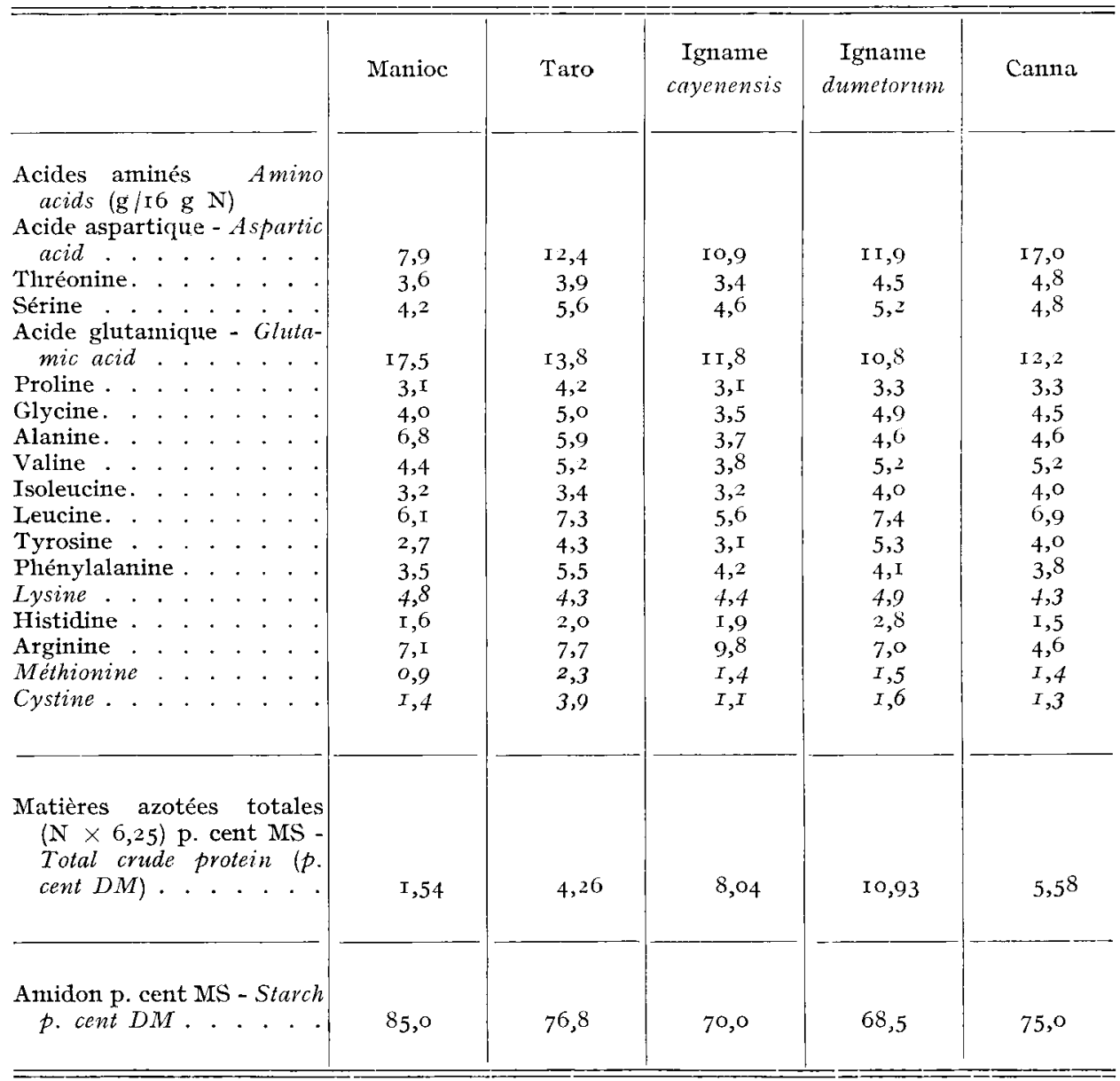

Dans chaque aliment granulé, l'état de l'amidon a été défini par des examens de microscopie photonique et sa sensibilité à une alpha-amylolyse in vitro a été de nouveau déterminée.

\section{III. - Tests biologiques}

Ces tests concernent le contrôle de la croissance des animaux, les bilans digestifs d'amidon et la rétention azotée. Le critère d'efficacité alimentaire retenu est l'indice de consommation, c'est-à-dire la quantité d'aliment consommé rapportée au gain de poids. 
TABLEAU 2

Composition centisémale des régimes

Composition of diets (p. cent)

\begin{tabular}{|c|c|c|c|c|c|}
\hline $\begin{array}{l}\text { Régimes } \\
\text { Diets }\end{array}$ & $\begin{array}{l}\text { Manioc } \\
\text { Cassava }\end{array}$ & $\begin{array}{l}\text { Taro } \\
\text { Taro }\end{array}$ & $\begin{array}{c}\text { Igname } \\
\text { dumetorum } \\
\text { Yam } \\
\text { dumetorum }\end{array}$ & $\begin{array}{c}\text { Igname } \\
\text { cayenensis } \\
\text { Yam } \\
\text { cayenensis }\end{array}$ & Canna \\
\hline 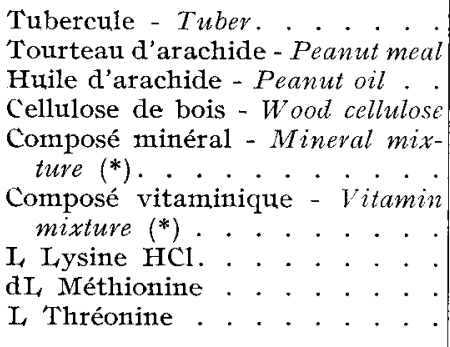 & $\begin{array}{r}57,2 \\
34,8 \\
4,0 \\
0,5 \\
3,0 \\
0,5 \\
0,50 \\
0,34 \\
0,19\end{array}$ & $\begin{array}{r}6 \mathrm{I}, \mathrm{O} \\
3 \mathrm{I}, 5 \\
4,0 \\
-- \\
3,0 \\
0,5 \\
0,39 \\
0,15 \\
0,11\end{array}$ & $\begin{array}{l}70,0 \\
22,0 \\
4,0 \\
0,5 \\
3,0 \\
0,5 \\
0,35 \\
0,35 \\
0,04\end{array}$ & $\begin{array}{l}3,0 \\
0,5 \\
0,34 \\
0,26 \\
0,11\end{array}$ & $\begin{array}{l}62,0 \\
30,5 \\
4,0 \\
- \\
3,0 \\
0,5 \\
0,40 \\
0,20 \\
0,12\end{array}$ \\
\hline 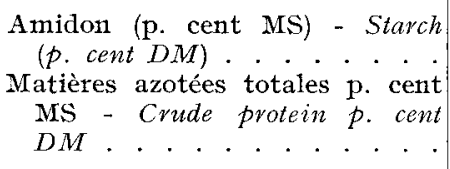 & I 8,0 & I 8,0 & 49,0 & I 8,0 & I 7,6 \\
\hline
\end{tabular}

(*) Formule décrite dans (see) Szylit, Delort-Laval et Borgida(1974).

\section{a) Croissance}

Les gains de poids de coquelets (souche I-99) placés en cages individuelles sont mesurés entre trois et six semaines d'âge. Les poulets sont mis en lots comparables sur la base du poids vif et du gain de poids pendant les deux semaines qui précèdent l'essai. Des difficultés rencontrées dans l'approvisionnement en tubercules ont imposé le nombre réduit de sept animaux par régime, la durée abrégée du test, trois semaines au lieu de quatre habituellement, et n'ont pas permis d'effectuer les bilans d'amidon dans le cas des poulets nourris au taro.

\section{b) Bilans d'amidon et d'azote}

A la suite du test de croissance, trois poulets de chaque lot (sauf le lot nourri au taro) sont maintenus trois jours en cage individuelle et reçoivent une même quantité d'aliment. Les refus sont pesés. Les excreta sont 1yophilisés et analysés pour leur teneur en amidon et azote, selon les méthodes déjà décrites pour l'analyse de 1'aliment. 
$55^{2}$ O. SZYLIT, L. P. BORGIDA, H. BEWA, R. CHARBONNIÈ,RE, J. DELOR'T-I,AVAL

dans le tubercule seul in the tuber alone
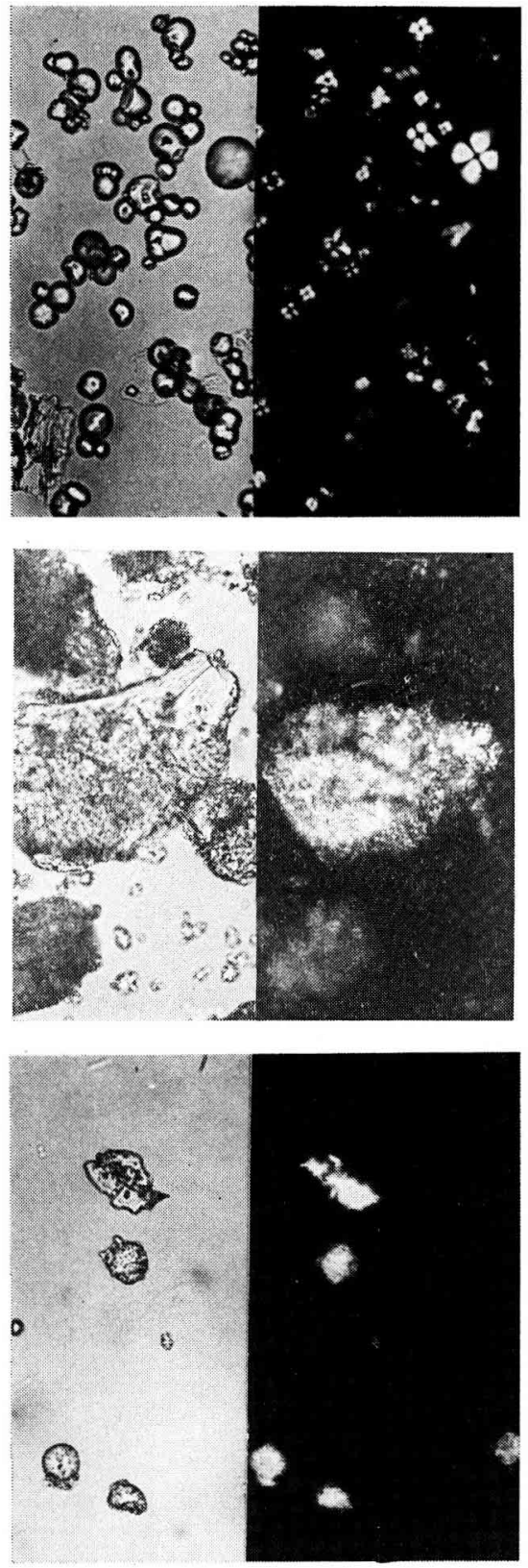

lumière blanche normal light lumière polarisée polarized light

dans le régime

in the diet
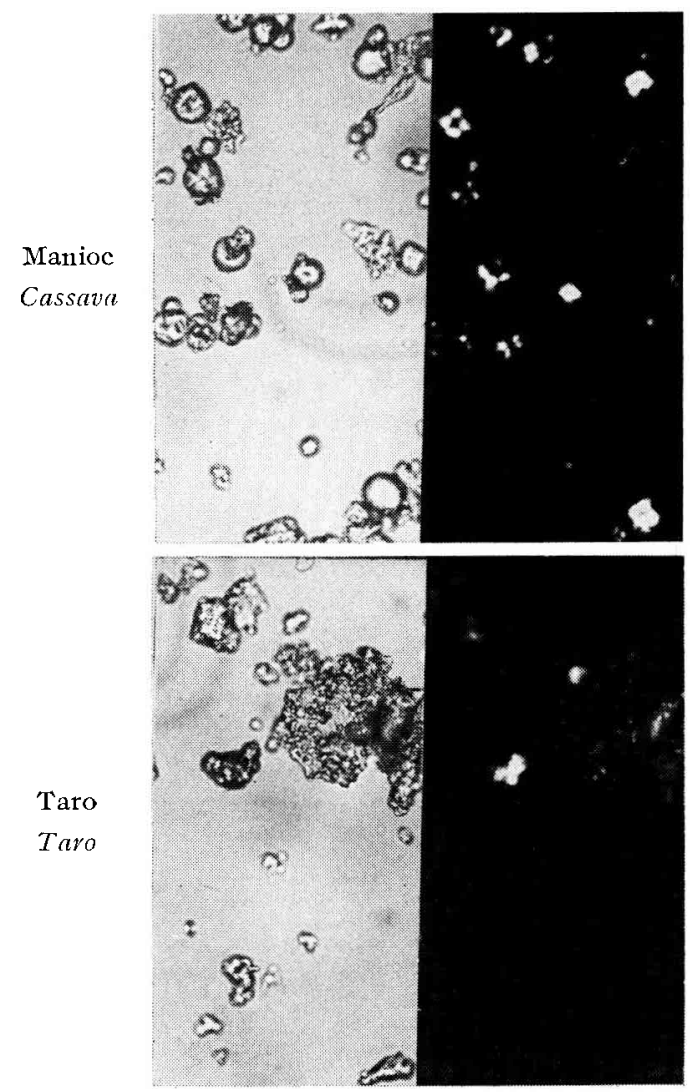

\section{E}

(3)

Igname dumetorum $\underset{\text { Yam }}{\text { dumetorur }}$

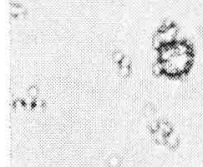

lumière blanche normal light

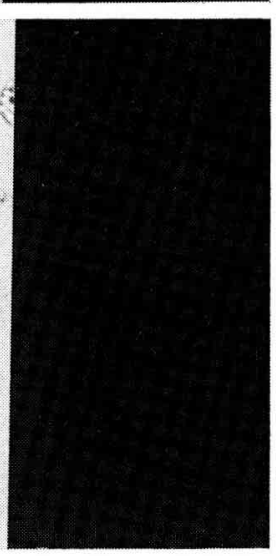

lumière polarisée polarized light

Fig. Ia. - Examen microscopique de grains d'amidon de tubercules de type $A$. Photomicrographs of starchs granules in tuber of the A type. 


\section{Résultats}

\section{I. - Caractéristiques des grains d'amidon}

\section{a) Aspect morphologique}

Suivant l'origine des tubercules, les tailles des grains d'amidon varient notablement (fig. I $a$ et $\mathrm{I} b$ ). Les plus petits sont sphériques et s'observent chez le taro (diamètre inférieur au micromètre) et chez l'igname dumetorum ( $\mathrm{I}$ à $2 \mu \mathrm{m}$ ). Souvent ces petits grains sont groupés dans des cellules. Les grains d'amidon de manioc

dans le tubercule seul

in the tuber alone
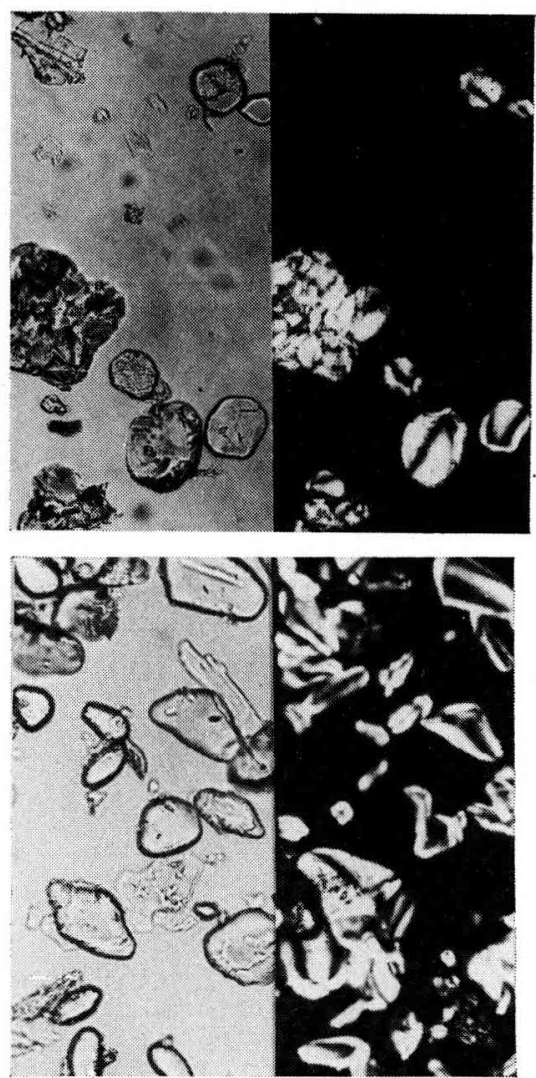

lumière blanche normal light
Iumière polarisée polarized light
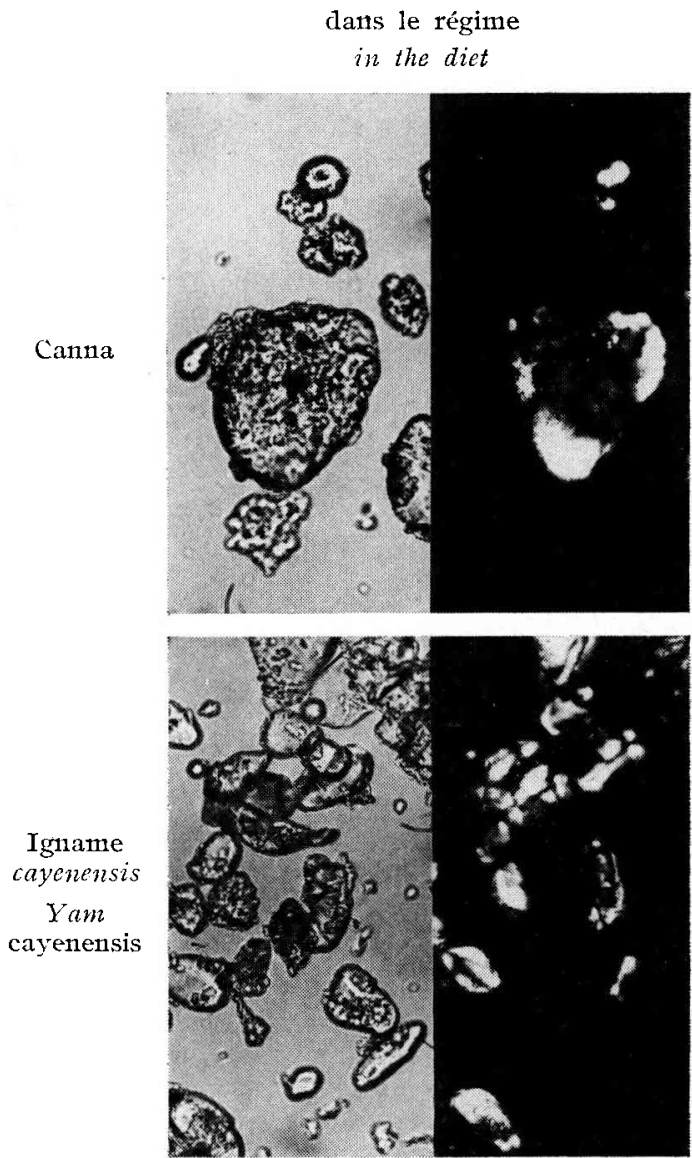

lumière blanche lumière polarisée normal light polarized light

FIG. $x$ b. - Examen microscopique des grains d'amidon des tubcrules de type $B$. Photomicrographs of starch granules in tuber of the B type 
sont sphériques ou ovales, les formes étant souvent tronquées; ils sont de taille moyenne (I $2 \mu \mathrm{m})$ et présentent tous un hile central. Ceux de l'igname cayenensis se distinguent par leur forme ovoïde, avec un hile excentré, et leur taille importante (75 $\mu \mathrm{m}$ environ); l'irrégularité de la croix de polarisation à laquelle ils donnent lieu témoigne d'une surface de grain à nombreuses arêtes. Les grains d'amidon de Canna sont de forme arrondie et régulière et de taille également importante (6o $\mu \mathrm{m})$.

L'examen microscopique des aliments granulés à base de taro ou d'igname dumetorum montre que la granulation fait éclater les parois cellulaires englobant les grains d'amidon. Dans le cas des granulés à base de manioc, d'igname cayenensis ou de Canna, elle entraîne un gonflement et une déformation des grains d'amidon, particulièrement ceux du Canna dont l'examen en lumière polarisée montre nettement que certains d'entre eux sont très endommagés.

\section{b) Organisation cristalline}

Les amidons d'igname cayenensis et de Canna donnent lieu à un même spectre de diffraction de rayons $\mathrm{X}$, le spectre de type $\mathrm{B}$ (fig. 2a). Par contre, ceux de manioc, de taro et d'igname dumetorum donnent lieu à des spectres de type A (fig. $2 b$ ).

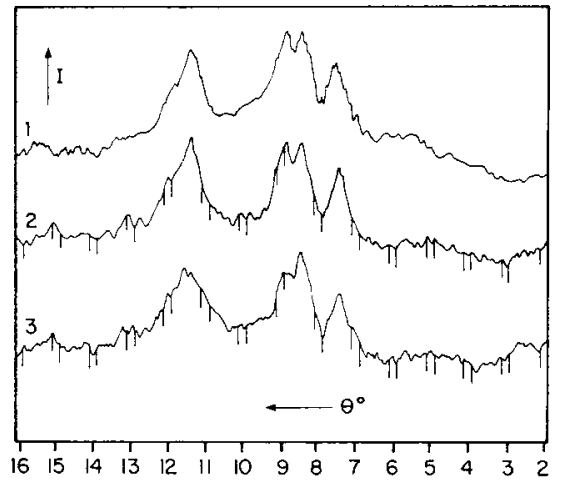

Amidon de type A Type A starches.

I. Manioc (Cassava).

2. Taro (Taro).

3. Igname dumetorum ( Tam dumetorum).

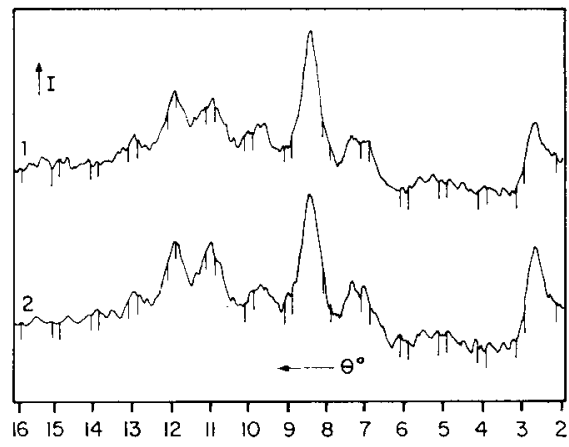

Amidon de type $\mathrm{B}-\mathrm{B}$ type starches.

I. Igname cayenensis - Yam cayenensis.

2. Canna.

FIG. 2. - Spectres de diffraction aux rayons $X$ des amidons de tubercules $X-r a y$ diffraction patterns of tuber starches.

\section{c) Teneur apparente en amylose}

Les teneurs apparentes en amylose des amidons de l'igname cayenensis et du Canna sont très voisines et relativement élevées (respectivement 26,9 et 26,7 p. cent de la masse d'amidon sec); celles des amidons de l'igname dumetorum et du taro sont toutes deux de même ordre, mais nettement plus basses que les précédentes (I0,o et $8,8 \mathrm{p}$. cent). La teneur en amylose de l'amidon de manioc est intermédiaire (I6,8 p. cent). 
d) Sensibilité de l'amidon dans le tubercule et dans l'aliment granulé à une alphaamylolyse bactérienne in vitro

Par ordre de sensibilité décroissante à tune alpha-amylolyse bactérienne pratiquée in vitro sur les tubercules, les amidons considérés se classent comme suit: manioc, igname dumetorum, Canna, taro, igname cayenensis (fig. 3).

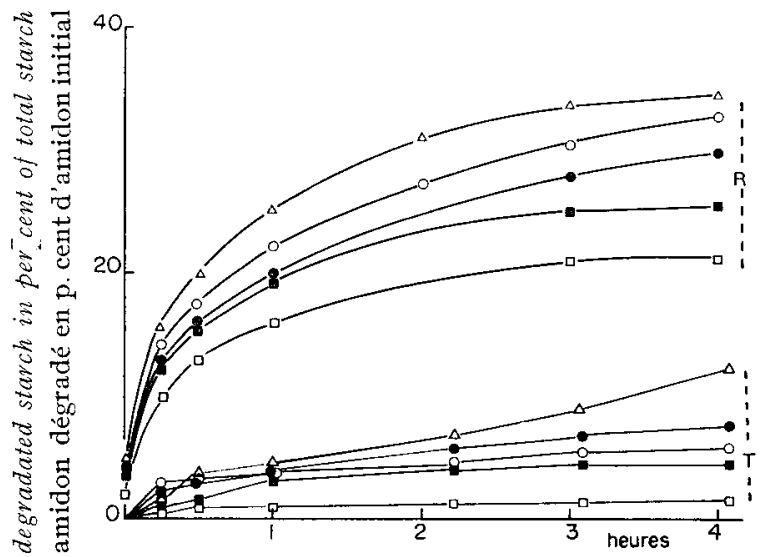

FIG. 3. - a-amylolyse in vitro des amidons de tubercules.

In vitro $\alpha$-amylolysis of tuber starches.

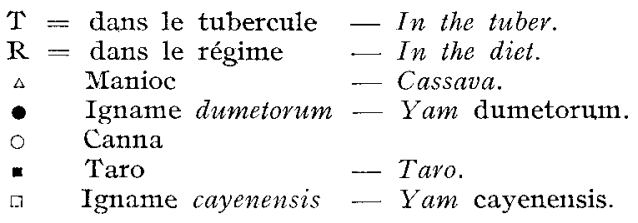

\section{TABIEAU 3}

Quantité d'amidon degradé in vitro en 4 heures d'hydrolyse par l'alpha-amylase de B. subtilis exprimée en $p$. cent de la teneur en amidon du tubercule

In vitro break down of tubers starches ( $p$. cent) reithin a 4 hours hydrolysis by $\alpha$-amylase of $\mathrm{B}$. subtilis

\begin{tabular}{c|c|c|c|c|c}
\hline $\begin{array}{c}\text { Tubercule } \\
\text { Tuber }\end{array}$ & $\begin{array}{c}\text { Manioc } \\
\text { Cassava }\end{array}$ & $\begin{array}{c}\text { Igname } \\
\text { dumetorum } \\
\text { Yam dumetorum }\end{array}$ & Canna & Taro & $\begin{array}{c}\text { Igname } \\
\text { cayenensis } \\
\text { Yam cayenensis }\end{array}$ \\
\hline Isolé - Alone..... & $\mathrm{I} 3$ & 8 & 6 & 5 & \\
\hline $\begin{array}{c}\text { Incorporé au régime - In } \\
\text { the diet. . . . . }\end{array}$ & 34 & 30 & 32 & 25 & $2 \mathrm{r}$ \\
\hline
\end{tabular}


Dans les aliments granulés, ces amidons apparaissent notablement plus sensibles à l'action amylolytique et on observe une inversion de classement entre 1'amidon de Canna et celui de l'igname dumetorum (fig. 3). Une appréciation quantitative des altérations subies par les grains d'amidon au cours du traitement de granulation des aliments est fournie par la comparaison des taux de dégradation des amidons dans les tubercules et dans les aliments granulés, estimés par les quantités de glucides alcoolosolubles libérés en quatre heures d'hydrolyse (tabl. 3).

\section{II. - Efficacité nutritionnelle des tubercules}

\section{a) Croissance des animaux}

Les courbes de croissance des poids moyens des animaux en fonction de la durée des essais montrent (fig. 4) qu'à la fin de la deuxième semaine les gains de poids avec les régimes à base de manioc, de taro, d'igname dumetorum et de Canna

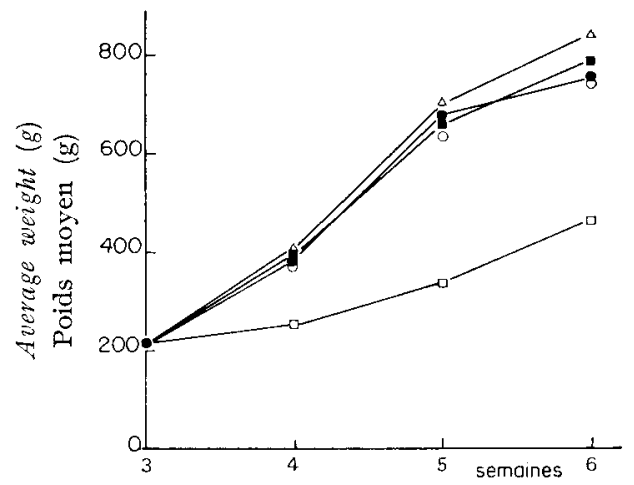

FIG. 4. - Evolution du poids moyen des coquelets

entre 3 et 6 semaines $(\mathrm{n}=7)$, en fonction du régime.

Average weight gain of chickens between 2 and 6 weeks $(\mathrm{n}=7)$ according to diets.

Tubercules de base des régimes -. Diets containing.

$\triangle$ Manioc -Cassava.

- Taro - Taro.

- Igname dumetorum - Yam dumetorum.

- Canna

- Igname cayenensis - Yam cayenensis.

sont de même ordre, soit une augmentation du poids initial de 200 p. cent. Par contre, avec le régime à base d'igname cayenensis, le gain de poids n'est que d'environ $5^{\circ} \mathrm{p}$. cent. A la fin de la troisième semaine, les poids moyens se rapportant aux quatre premiers régimes diffèrent un peu, mais les écarts n'atteignent pas Io p. cent. Avec le dernier régime, la croissance reste mauvaise, le gain de poids étant de 1 'ordre de Ioo $\mathrm{p}$. cent contre au moins $25^{\circ} \mathrm{p}$. cent avec les autres régimes, par rapport au poids initial.

Même lorsque les croissances sont de même ordre, la consommation des régimes est variable, plus élevée avec ceux à base de taro ou de Canna qu'avec ceux à base d'igname dumetorum ou, surtout, de manioc. Le régime à base d'igname cayenensis est de beaucoup le moins consommé (tabl. 4). 
TABIEAU 4

Croissance des poulets et efficacité alimentaire des régimes ( $m \pm s m)$

Growth of chickens and feed efficiency of diets

\begin{tabular}{|c|c|c|c|}
\hline & $\begin{array}{c}\text { Gain de poids } \\
\text { g/jour (GP) } \\
\text { Weight gain (g/day) }\end{array}$ & $\begin{array}{c}\text { Consommation } \mathrm{g} \\
\text { MS/jour (C) } \\
D M \text { intake }(g / d a y)\end{array}$ & $\begin{array}{l}\text { Indice de consom- } \\
\text { mation (C)/(GP) } \\
\text { Feed conversion ratio }\end{array}$ \\
\hline 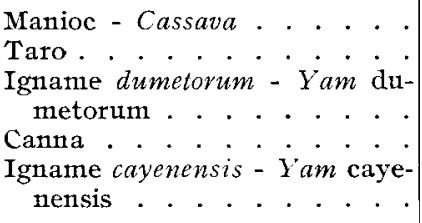 & $\begin{array}{l}4^{o, 0^{a}} \\
37,3^{b} \\
36,0^{b} \\
3^{6}, I^{b} \\
12,4^{c}\end{array}$ & $\begin{array}{l}82,3^{c} \pm 3,1 \\
99,5^{a} \pm 3,9 \\
83,4^{c} \pm 2,6 \\
91,3^{b} \pm 2,2 \\
63,6^{d} \pm 2,5\end{array}$ & $\begin{array}{l}2,10^{a} \pm 0,13 \\
2,70^{a} \pm 0,17 \\
2,38^{a} \pm 0,16 \\
2,53^{a} \pm 0,08 \\
6,64^{b} \pm 1,90\end{array}$ \\
\hline
\end{tabular}

Les moyennes accompagnées de la même lettre, ne diffèrent pas significativement $(\mathrm{P}<0,05)$. Means followed by the same letter are not significantly different $(P C<0,05)$.

Le régime à base de manioc est donc le mieux utilisé. En prenant pour référence son indice de consommation, ceux des autres régimes sont supérieurs de I2,8 p. cent avec l'igname dumetorum, 28 p. cent avec le taro et le Canna, I66 p. cent avec l'igname cayenensis.

TABI,EAU 5

Bilans digestifs de l'amidon et rétention azotée (moyennes journalièves pour 3 animaux)

Digestive balances of starch and nitrogen retention (daily means for 3 animals)

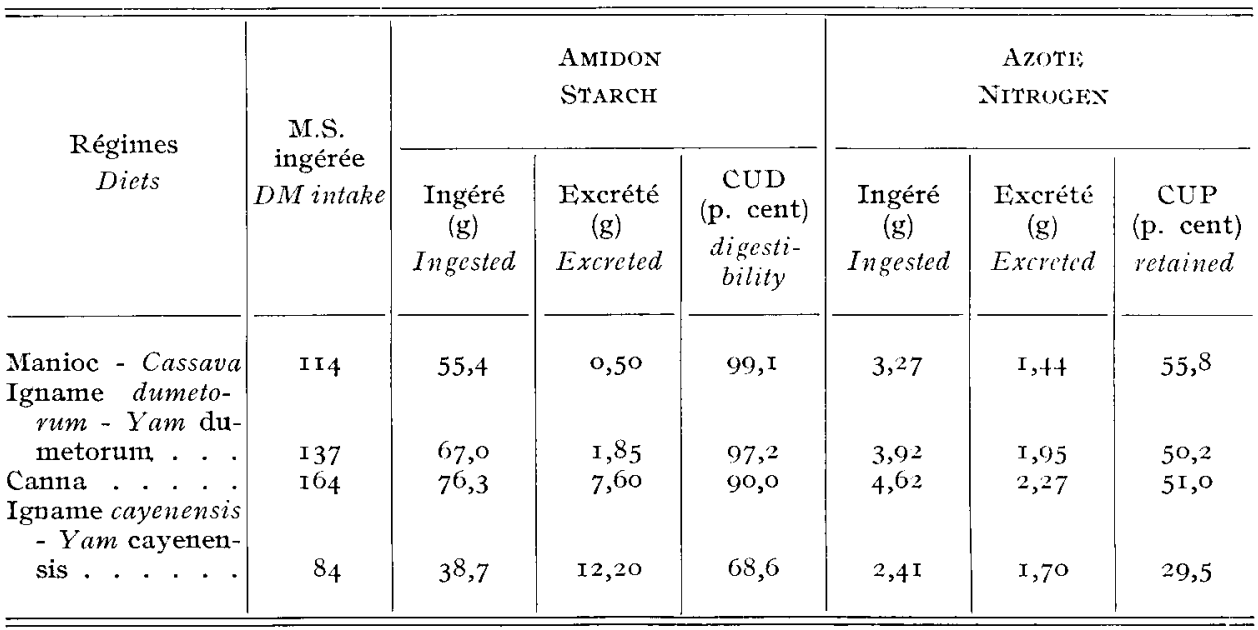


b) Digestion de l'amidon ct bilan azoté

Les bilans digestifs des amidons (tab. 5) indiquent que ceux de manioc et d'igname dumetorum sont à peu près totalement digérés, alors que l'amidon de Canna ne l'est qu'à go p. cent. Quant à l'igname cayenensis, 30 p. cent de son amidon est indigestible.

Le coefficient d'utilisation pratique de l'azote varie suivant les régimes (tabl. 5) : il est relativement élevé avec le manioc (56 p. cent), sensiblement plus faible avec le Canna et l'igname dumetorum (50-5I p. cent), et particulièrement bas avec l'igname cayenensis (29 p. cent).

\section{Discussion et conclusion}

Bien que les amidons des tubercules de manioc, d'igname dunctorum, de taro et de Canna n'aient pas les mêmes caractéristiques physiques, les régimes constitués avec ces tubercules donnent dans nos conditions expérimentales des réponses nutritionnelles similaires si l'on considère uniquement la vitesse de croissance. Toutefois, des différences apparaissent si l'on s'intéresse, à l'efficacité alimentaire de chaque régime qui tient compte de la consommation de l'aliment, ou encore au coefficient d'utilisation de l'amidon lié à sa sensibilité aux alphaamylases (tabl. 6). Quant au régime à base de tubercules d'igname cayenensis, il donne des résultats nettement moins bons, alors que le taux de dégradation amylo-

TABLEAU 6

Comparaison entre différents critères physico-chimiques et la valeur nutritionnelle de l'amidon Comparison between various physico-chemical criteria and nutritional value of starch

\begin{tabular}{|c|c|c|c|c|c|c|}
\hline \multirow{2}{*}{$\begin{array}{l}\text { Tubercule } \\
\text { Tuber }\end{array}$} & \multicolumn{2}{|c|}{$\begin{array}{c}\text { Valeur } \\
\text { nutritionnelle } \\
\text { Nutritional value }\end{array}$} & \multicolumn{4}{|c|}{$\begin{array}{l}\text { Caractéristiques structurales de l'amidon } \\
\text { Structural characteristics of starch }\end{array}$} \\
\hline & $\begin{array}{l}\text { Indice de } \\
\text { consom- } \\
\text { mation } \\
\text { Feed conver- } \\
\text { sion ratio }\end{array}$ & $\begin{array}{l}\text { CUD } \\
\text { Amidon } \\
\text { Starch } \\
\text { digesti- } \\
\text { bility }\end{array}$ & $\begin{array}{c}\text { Taille } \\
(\mu \mathrm{m}) \\
\text { des grains } \\
\text { Size of } \\
\text { granules }\end{array}$ & $\begin{array}{c}\text { Type } \\
\text { de } \\
\text { spectre } \\
\text { Spectrum } \\
\text { type }\end{array}$ & $\begin{array}{c}\text { Taux } \\
\text { d'amylose } \\
\text { Amylose } \\
\text { level }\end{array}$ & $\begin{array}{l}\text { Amidon } \\
\text { facilement } \\
\text { attaquable } \\
\text { Easily atta- } \\
\text { ched starch }\end{array}$ \\
\hline $\begin{array}{l}\text { Manioc - Cassava } \\
\text { I. dumetorum-" } \\
\text { Yam dumeto- } \\
\text { rum . . . . } \\
\text { Canna. . . . } \\
\text { Taro - Taro . . } \\
\text { I. cayenensis-Yam } \\
\text { cayenensis. . . }\end{array}$ & $\begin{array}{l}2,38 \\
2,53 \\
2,70 \\
6,64\end{array}$ & $\begin{array}{l}97,2 \\
90,0 \\
-\end{array}$ & $\begin{array}{r}r-3 \\
60 \\
I\end{array}$ & $\begin{array}{l}\mathrm{A} \\
\mathrm{B} \\
\mathrm{A} \\
\mathrm{B}\end{array}$ & $\begin{array}{r}13,4 \\
26,9 \\
8,8 \\
\\
26,7\end{array}$ & $\begin{array}{c}8 \\
6 \\
5 \\
\mathrm{I}, 5\end{array}$ \\
\hline
\end{tabular}


lytique in vitro de l'amidon de ces tubercules est voisin de celui de 1'amidon des tubercules de taro pourtant beaucoup mieux utilisés in vivo.

Pour tenter de relier l'efficacité alimentaire d'un amidon aux critères physicochimiques retenus pour le caractériser, examinons séparément l'influence éventuelle de chaque paramètre :

\section{a) Taille des grains}

Si l'on se limite à la comparaison des valeurs nutritionnelles des tubercules d'ignames, nos résultats s'accordent avec ceux de RASPER (r969), qui trouve que la digestibilité in vitro du grain d'amidon est d'autant plus élevée que sa taille est plus petite. En effet, l'amidon de l'igname dumetorum, à grains à $\mathbf{I} \mu \mathrm{m}$ est beaucoup mieux digéré que celui de l'igname cayenensis, à grains de $70 \mu \mathrm{m}$. Dans le même ordre d'idées, MASon et PALMER (I973) trouvent que le grain d'amidon de l'igname alata, voisin par la taille de celui de l'igname cayenensis (RASPER et Coursey, I967) n'est pas digestible par le rat. D'at tre part, le grain d'amidon de Canna edulis, décrit par KAY (1973) comme étant de grande taille (145 $\mu \mathrm{m}$ ), est peu digestible in vitro (3 p. cent) sous l'action de la pancréatine. La variété que nous avons étudiée, constituée de grains deux fois plus petits, est beaucoup mieux digérée, tant in vitro qu'in vivo.

Cependant, la différence entre les valeurs nutritionnelles des aliments à base d'igname cayenensis et de Canna, par ailleurs comparables par l'organisation de leur structure cristalline (spectres de type B), ne semble pas explicable par le seul facteur taille. Entre autres facteurs susceptibles d'intervenir, on peut encore invoquer l'étendue des distributions de tailles des grains d'amidon, la forme de ces grains qui peut, semble-t-il, conditionner la pénétration de l'enzyme (GALLANT et al., I974), puisque les amidons de formes peu régulières (igname cayenensis, amylo-maïs) apparaissent comme les moins digestibles.

\section{b) Organisation cristalline}

Les amidons ayant une organisation cristalline à spectre de type $\mathrm{A}$ (manioc, igname dumetorum, taro) sont tous bien digérés, alors que ceux dont 1'organisation cristalline est à spectre de type B (Canna, igname cayenensis) sont moins bien utilisés, mais avec des efficacités variables, le coefficient d'utilisation digestive passant de go pour le Canna à 69 pour l'igname cayenensis.

Ces résultats relatifs à des amidons de tubercules sont à rapprocher de cenx obtenus avec des amidons de céréales : l'amidon de maïs normal, à spectre de type $\mathrm{A}$, est digestible à $99 \mathrm{p}$. cent, alors que celui d'amylomaïs, à spectre de type B, ne l'est qu'à 76 p. cent (Szylit, Delort-LAval et Borgida, I974). Ces concordances incitent à poursuivre plus avant l'étude de la relation entre organisation cristalline et utilisation digestive.

\section{c) Teneur en amylose}

Les amidons les moins digestibles (Canna, igname cayenensis) sont précisément ceux ayant les teneurs en amylose les plus élevées, correspondance que l'on retrouve dans le cas d'un amylomaïs. Cependant, cette relation ne saurait être 
généralisée puisque l'amidon de pomme de terre est également peu digestible par le poulet alors que sa teneur en amylose est seulement de l'ordre de I 9 p. cent pour les espèces les plus communément utilisées.

\section{d) Sensibilité aux amylases}

L'efficacité nutritionnelle des tubercules et le coefficient d'utilisation digestive de leur amidon augmentent avec la proportion d'amidon facilement dégradable in vitro par l'alpha-amylase bactérienne. Par contre, dans le cas d'amidons de maïs à différentes teneurs en amylose, c'est le test d'amylolyse effectué avec un mélange d'alpha-amylases bactérienne et pancréatique qui rend le mieux compte de leur digestibilité et de leur efficacité alimentaire (SzyLIT, DEI,ORT-LAVAL et BORGIDA, I974).

Le pressage à sec intervenant dans la fabrication de l'aliment modifie de façon notable 1'accessibilité in vitro de 1'amidon à 1' $\alpha$-amylase bactérienne, en accord avec les conclusions d'autres auteurs sur les amidons de céréales et de pommes de terte (Mercier, Charbonnière, Guilbot, ig68).

Néanmoins, les résultats de l'amylolyse in vitro doivent être considérés avec réserve. En effet, alors que nos données concordent avec celles de FAviFR (I969), la même méthode ayant été utilisée, celles de RASPER ( 1969 ) font apparaître pour le manioc et le taro une inversion de leurs vitesses d'hydrolyse déterminées en utilisant des concentrations plus faibles en enzymes et en estimant, par mesure de pouvoir réducteur, les quantités de glucides formés en vingt heures. Par ailleurs, le comportement d'un amidon à une amylolyse in vitro n'est pas en relation simple ni avec la taille des grains, ni avec le type de son organisation cristalline.

Avec les tubercules considérés ici, ce test a donné des résultats en accord avec des mesures de disponibilité de leurs amidons pour la croissance in vitro de la microflore du rumen (DURAND et al., I975). Ces mesures attribuent la même valeur au manioc qu'à l'igname dumetorum, une valeur intermédiaire plus faible au canna, et une valeur beaucoup plus faible à l'igname cayenensis.

D'un point de vue uniquement nutritionnel, on constate que l'utilisation digestive de l'amidon conditionne en partie l'utilisation des protéines. Elle est particulièrement mauvaise avec l'igname cayenensis (29 p. cent) en raison de la faible consommation de 1'aliment et de son utilisation digestive médiocre, vraisemblablement responsable d'une excrétion accrue d'azote métabolique. Il est d'ailleurs connu que l'utilisation des protéines varie avec la nature des glucides (HARPER, Katayama et Jélinek, I952; Zeiter, Chari,ET-Lery, IOelort-Laval, I966). Ainsi la quantité d'azote fécal excrétée par le rat (Mason, PALMER, I973) n'est pas modifiée en substituant à l'amidon de riz d'autres amidons parfaitement digestibles (manioc, maïs). Par contre, l'amidon d'igname alata, que l'on peut apparenter à celui d'igname cayenensis (RASPER et COURSEY, I967), par la taille de ses grains et son spectre de diffraction, provoque une augmentation considérable de l'azote fécal métabolique associée à une augmentation parallèle de l'acide diaminopimélique, qui est un acide aminé spécifiquement bactérien (MAson et PALMER, I973). La présence d'amidon résiduel non digéré dans les premières parties de 1'intestin entraînerait dans la partie distale un développement de la population bactérienne qui fixe l'azote disponible aux dépens de l'animal.

En conclusion, aucun des paramètres physico-chimiques considérés, taille de grain, organisation cristalline, teneur en amylose, sensibilité in vitro à une alphaamylolyse, ne peut constituer à lui seul un critère de classement de la valeur nutri- 
tionnelle d'un amidon. Tout au plus, avec les amidons étudiés, une petite taille de grain, un spectre de type $\mathrm{A}$, une faible teneur en amylose, une bonne digestibilité in vitro sont autant de caractères laissant présumer une bonne valeur nutritionnelle sans qu'ils constituent cependant des critères absolus. Néanmoins, l'étude des tubercules tropicaux nous permet de nuancer les résultats obtenus à partir des amidons de maïs à taux d'amylose variés : les amidons de type $B$ sont toujours moins digestibles in vivo que ceux de type A, mais l'efficacité alimentaire du tubercule est plus spécialement en liaison avec la vitesse d'amylolyse in vitro de l'amidon. Une étude poursuivie en utilisant des amidons extraits nous permettra de vérifier plus précisément l'effet spécifique de l'amidon.

En Europe, on ne connaît guère qu'un tubercule, la pomme de terre dont la valeur alimentaire est médiocre à l'état cru. Par contre, certains tubercules tropicaux, sont susceptibles, comme les céréales, d'être digérés sans cuisson préalable. Jusqu'ici, seul le manioc et la patate douce (Ipomoea batatas) ont été bien étudiés. Il serait d'un grand intérêt économique de rechercher parmi les nombreuses variétés de tubercules des régions tropicales humides, celles, qui sont utilisables en alimentation animale. La connaissance des caractéristiques physicochimiques de leur amidon peut contribuer à cette sélection.

Accepté pour publication en aồt 1977.

\section{Remerciements}

Nous remercions M. VIROBEN (Laboratoire de Technologie des Aliments des Animaux) pour le dosage des acides aminés et Mme FARBER (Laboratoire de Technologie Alimentaire) pour les examens de microscopie.

Nous remercions M. GUIr BOT de ses conseils pour la rédaction de cet article.

\section{Summary}

Nutritional value, for the growing chicken

of five tropical amylaceous feeds in connection ieith some characteristics of their starch

The aim of this experiment on growing chickens was to estimate the feed efficiency of five tropical roots, according to the physico-chemical characteristics of their starch.

Starch of Cassava (Manihot utilissima), Taro (Colocassia antiquorum) and Yam dumetorum (Dioscorea dumetorum) is characterised by granules of small diameter (fig. I $a$ ) and a X-ray diffraction pattern of the A type (fig. 2), and is therefore similar to starch of cereals. Canna (Canna edulis) and Yam cayenensis (Dioscovea cayenensis) have much larger starch granules (60-75 $\mu)$ (fig. I b) with a high amylose level (26 p. cent) and a X-ray pattern (fig. 2) of the B type as potato starch.

In vitro breakdown of these starches in the presence of bacterial $\alpha$-amylase from $B$. subtilis decreases in the following order : Cassava, Yam dumetorum, Taro, Canna, Yam cayenensis (fig. 3). Three-weeks old chickens received for $2 \mathrm{r}$ days a diet based on peanut meal and tropical tubers (table 2). Very slight differences were observed with the first four diets concerning the average weight gain $(40,36,37$ and $36 \mathrm{~g}$ per day, respectively), but the daily food intake was different $(82,83,99$ and $91 \mathrm{~g}$ per day). Consequently the best feed efficiency was obtained with Cassava diet (fig. 4 and table IV). The poorest prowth ( $2 \mathrm{~g}$ per day) and the lowest feed intake $(64 \mathrm{~g}$ per day) was recorded with the diet containing Yam cayenensis.

Starches with X-ray diffraction pattern of the A type were all completely digested ( 98 p. cent); 
the digestibility of those of the $B$ type was lower: $90 \mathrm{p}$. cent for the Canna and $69 \mathrm{p}$. cent for Yam cayenensis. For the latter, this lead to reduction of the level of protein utilisation (table 5). Similar results have been already obtained with high amylose maize starch which has also a X-ray diffraction pattern of the B type.

In conclusion, none of the physico-chemical or enzymatic parameters studied can be used as sole grading criterium for the nutritional value of a given starch. However, a starch granule with a small diameter, a low amylose content and a X-ray diffraction pattern of the A type are all favourable factors contributing to a good apparent digestibility of starch. In addition, it appears that feed efficiency of a tuber is more especially related to the rate of the in vitro amylo1ysis of starch.

\section{Références bibliographiques}

Atrnkpahoun H., I972. Contribution à l'étude de la valeur nutritionnelle, pour le poulet de trois plantes tropicales; Manihot utilissima, Ipomoae batatas, Dioscorea cayenensis. Thèse Doctorat $3^{\mathrm{e}}$ cycle, Paris VI.

Baker F., NASR H., MORRICF F. et BRUCE J., I95o. Bacterial breakdown of structural starch products of the digestive tract of ruminants and non ruminants mammals. J. Path. Bact., L.XII, 6I $7-638$.

BEMILLER J. N., I964. Iodimetric determination of anylose in "Methods in carbohydrate chemistry ", t. IV, R. I. Whistı.er., Ed Academic Press. - New York, Londres, I65-I69.

Charbonniere, R., Mercier C., Torlier M. T. et GuilboT A., ig68. Étude diffractométrique des amidons de maîs à teneur variable en amylose. Stärke, $20,75-78$.

Durand M., GUeguen I., Prifio F., Szyiti O., I975. Effect of source of energy and minerals on microbial protein synthesis in the rumen using labelled sulfur as indicator. In "Tracers studies on non-protein nitrogen for ruminants $I I$ ", I.A.E.A., Vienna, 29-42

EWERS, 1972. Dosage de 1'amidon, méthode polarimétrique. J.O. C.E.E., 15, I, I23, 7-9.

FAvier J. C., r969. Étude de la digestibilité in vitro de l'amidon de diverses plantes alimentaires du Sud Cameroun. Influence des transformations technologiques sur l'amidon du manioc. Ind. Agri., Alim. 86, 9-I I.

GALLANT D., I974. Contribution à l'étude de la structure et de l'ultrastructure du grain d'amidon. Thèse de Doctorat Sciences Physiques, Paris.

Gallant D., Mercier C., Guilbot A., I972. Electron microscopy of starch granules modified by bacterial $\alpha$-amylase. Ceveal chem., 49, 403-354-365.

HaRper A. E., KaTAYama M. C. et JeLINEK B., 1952. The influence of dietary carbohydrates on levels of amino acids in the feces of the white rat. Can. J. Med. Sci., 30, 578-592.

KAy D., 1973. Roots Crop. Tropical crop and product. Digest no 2 . London tropical product inst., 245 .

LANGWOR'THY C. F, and DE CEL H. J., I920. Digestibility of raw corn, potato and wheat starches. J. Biol. Chem., 4, 2-27.

MAson V. C., PALMER R., I973. The influence of bacterial activity in the alimentary canal of rats on faecal nitrogen excretion. Acta Agric. Scand., 23, I4 I-I $5^{\circ}$.

Masson M. J., I954. Microscopic studies of the gut flora of the hen, with special reference to the breakdown of starches. Ioth World's Poult. Congr., Edinburg, Io5-I II.

MERCIER C., I968. Contribution à l'étude de la structure du grain d'amidon aux moyens de méthodes physiques et enzymatiques. Thèse Doctorat d'État-ès-Sciences, C.N.R.S., AO 24 I3.

Mercier C., Charbonniere R. et Guilbo' A., i968. Influence d'un traitement par pression sur la structure granulaire de différents amidons et sur leur sensibilité aux enzymes. Stärke, 20, 6-II.

MoORE S., Spackmann D. H. et Stein N. M., I958. Chromatography of amino acids on sulfonated polystyrene resins. Anal. chem., 30 (7), i 85 -I r9o.

N.A.R., I974. Annotated bibliography no 42. Cassava. Commonwealth Bureau of Nutrition, Bucksburn Aberdeen (England), 23 pp., I30 réf.

Palissf, M. et Baratou J., I973. Le manioc et les patates douces, matières premières glucidiques pour le poulet de chair. J. Rech. Av. Cunéi., 4, C 1 , I65-I67.

RASPER V., I969. Investigations on starches from major starch crops grown in Ghana. II. Swelling and solubility patterns: amyloclastic susceptibility. J. Sci. Fd. Agric., 20, 642-646. 
Rasper V. et COURSey D. G., 1967. Properties of starches of some West African Yanı. $J$. Sci. Fid. Agric., 18, 240-244.

SPLitTOESSER W. E., MARTIN F. W. et RhoDes A. M., i973. The amino acid composition of five species of yams (Dioscorea). J. Amer. Soc. Hort. Sci., 98 (6), 563-567.

SQuiss R. I. et WYLD M. K., I95I. Effect of yuca meal in baby chick rations. Turvialba, 1, 298-299.

SZABUNIEWICZ M., 1953. Note sur quelques cultures fourragères au Katanga dans la région de Jadotville, Kolwezi et des Biano; Canna edulis. Bull. agric. Congo Belge, 44. (3), 604-612.

Szylir O., Delort-Lavai, J. et Borgida L. P., I974. Dégradation dans le jabot du coq et efficacité d'amidons de maïs à différents taux d'amylose sur la croissance du poulet. Ann. Zootech., 23, 253-265.

TABoyoyong T. T., 1935. The value of Cassava refuse meal in the ration for growing chick. Philippine Agric., 24, 509-518.

TORRes A. P., I935. A raspa da mandioca no alimentaçao dos gallinas. Ann. Esc. Sup. Agric. Luiz Querioz- Piracicaba, 3, 329-338.

VoGi H. et STUTE K., 1963. Prüfung von Tapiokamehl in Geflügelmast-Alleinfutter bei Beginn der Verabreichung in verschiedenen Altersstufen. Avchiv. tür geflugelkunde, 27, $473-482$.

Zelter S. Z., Chardet-Lery G. et DelorT-Laval J., ig66. Influence de la fécule crue et cuite de pomme de terre sur les dépenses azotées métaboliques et endogènes et sur la valeur nutritionnelle d'un mélange équilibré de protéines chez le porc en croissance. C. R. Acad. Agric. France, 567-573. 